\title{
Measurement of aerosol optical extinction using diode laser cavity ringdown spectroscopy
}

\author{
LIU YingDi \& ZHANG JingSong* \\ Department of Chemistry and Air Pollution Research Center, University of California, Riverside, CA 92521, USA
}

Received December 30, 2012; accepted March 7, 2013; published online May 14, 2013

\begin{abstract}
Accurate measurement of optical extinction of atmospheric aerosols is important for quantifying the direct climate effects of aerosols. A portable cavity ringdown spectrometer utilizing a modulated multimode blue diode laser (linewidth $\sim 0.2 \mathrm{~nm}$ ) is developed to measure the aerosol optical extinction. Laboratory generated ammonia sulfate particles $(<1 \mu \mathrm{m}$ in diameter $)$ are characterized, with good agreements between the experimental measurements and Mie theory calculations. An optical extinction detection sensitivity of $0.24 \mathrm{Mm}^{-1}(1 \sigma)$ is achieved. Measurements of ambient aerosols are also carried out. This study demonstrates the feasibility of a compact, multimode diode laser cavity ringdown spectrometer for sensitive measurements of the optical extinction of atmospheric aerosols.
\end{abstract}

aerosol, optical extinction, spectroscopy

Citation: Liu Y D, Zhang J S. Measurement of aerosol optical extinction using diode laser cavity ringdown spectroscopy. Chin Sci Bull, 2013, 58: 2440-2446, doi: $10.1007 / \mathrm{s} 11434-013-5825-\mathrm{z}$

Atmospheric aerosols interact with solar radiation by extinction of light, which includes both scattering and absorption. In general, the atmospheric aerosols absorb weakly that their extinction is mainly due to scattering. The atmospheric aerosols can directly influence the climate by heating up the atmosphere aloft on one hand, and by reducing surface irradiance on the other hand. They can also alter the properties of clouds and thus indirectly influence the climate in the atmosphere [1].

When a light beam illuminates on aerosol particles, a portion of the light beam is scattered and absorbed by the particles; thereby the intensity of the light beam is attenuated along the optical axis. The intensity of light that traverses the aerosols, $I$, is given by the Beer's law,

$$
I=I_{0} \exp \left(-\sigma_{\mathrm{e}} l\right),
$$

where $I_{0}$ is the intensity of incident light, $\sigma_{\mathrm{e}}$ is the aerosol extinction coefficient, and $l$ is the optical path length of the light through the aerosols. For mono-dispersed aerosols of a number density of $N$ particles per unit volume, $\sigma_{\mathrm{e}}$ is given

*Corresponding author (email: jingsong.zhang@ucr.edu) by [2]

$$
\sigma_{\mathrm{e}}=\varepsilon Q_{\mathrm{e}} N,
$$

where $\varepsilon$ is the geometrical cross section of the aerosol $\left(\pi d^{2} / 4\right.$ for a spherical particle with a diameter $d$ ), and $Q_{\mathrm{e}}$ is the particle extinction efficiency. The product of $\varepsilon$ and $Q_{\mathrm{e}}$ is the extinction cross section of the aerosol particle. For poly-dispersed aerosols, the optical extinction $\sigma_{\mathrm{e}}$ is the sum of the extinction cross section of individual aerosol particle, $\varepsilon Q_{\mathrm{e}}$.

$Q_{\mathrm{e}}$ is of theoretical and analytical importance. It is the ratio of the radiation power scattered and absorbed by a particle to the radiation power geometrically incident on the particle. The $Q_{\mathrm{e}}$ value depends on the particle refractive index $(n)$ and shape and size relative to the wavelength of light $(\lambda)$. The dependence on the particle size is expressed in a dimensionless size parameter $x$, which is the ratio of the particle diameter to the wavelength, $\lambda$, of the radiation light,

$$
x=\pi d / \lambda \text {. }
$$

As the optical extinction includes both scattering and absorption, the extinction efficiency of a particle is the sum 
of its scattering efficiency $Q_{\mathrm{s}}$ and absorption efficiency $Q_{\mathrm{a}}$,

$$
Q_{\mathrm{e}}=Q_{\mathrm{s}}+Q_{\mathrm{a}}
$$

Correspondingly, for mono-dispersed particles,

$$
\sigma_{\mathrm{e}}=\sigma_{\mathrm{s}}+\sigma_{\mathrm{a}},
$$

where $\sigma_{\mathrm{s}}$ and $\sigma_{\mathrm{a}}$ are the aerosol scattering coefficient and absorption coefficient, respectively.

While all aerosol particles scatter light, only those composed of absorbing material would absorb, and this absorption is described by the imaginary part of the refractive index. For non-absorbing particles, $Q_{\mathrm{e}}=Q_{\mathrm{s}}$. Scattering from larger spherical particles is explained by the Mie theory for an arbitrary size parameter $x$. For small size parameter $x$, the Mie theory reduces to the Rayleigh approximation (for scattering in the regime $x \ll 1)$. In the wavelength of visible light regime, for small spherical particles with $d<50 \mathrm{~nm}$, $Q_{\text {s }}$ can be calculated using the Rayleigh scattering theory. For larger spherical particles $(d>50 \mathrm{~nm}), Q_{\mathrm{s}}$ can be obtained by the Mie theory calculations.

Although the optical extinction of atmospheric aerosols has been measured for decades, a better understanding of the aerosol optical properties is still needed. In particular, in situ measurements of the extinction and other optical parameters of atmospheric aerosols are crucial, and these require real-time, accurate, and sensitive techniques that can detect the ambient aerosols and their rapid temporal and spatial changes. Recent years have seen a rapid rise in the use of cavity ringdown spectroscopy (CRDS), a sensitive and direct optical extinction technique, to determine the optical properties of both laboratory generated and atmospheric ambient aerosols [3-17].

CRDS was first developed by O'Keefe and Deacon [18] in 1988 and utilized for spectroscopic measurements of gas species absorption [19]. The CRDS technique measures the rate, rather than the magnitude, of extinction of light in a highly reflective optical cavity that contains the trace sample [20]. The rate of decay of the trapped light is determined by the total cavity loss and sample absorption. The net decay rate as a function of laser wavelength yields the absorption spectrum of the sample. With $\sim 10^{4}$ round trips of the trapped light, a long effective path $(\sim 10 \mathrm{~km}$ in a 1-m cavity) and thus high sensitivity are achieved. The CRDS technique is absolute, quantitative, and sensitive, and it is ideal for spectroscopic detection of atmospheric trace species. Many atmospheric species, such as $\mathrm{NO}_{2}$ [21-23], $\mathrm{NO}_{3}$ [24,25], $\mathrm{SO}_{2}$ [26] and peroxy radicals [27], have been measured by CRDS.

Sappey et al. [3] and Smith and Atkinson [4] were among the first research groups to apply CRDS in aerosol extinction measurements and quantify the extinction of the ambient atmospheric particles. Many more studies have been subsequently carried out to measure the optical extinction of aerosol particles using CRDS [6-10]. Most of these pervious works were based on using either a pulsed YAG laser, which is relatively bulky and limited to only 355,532 or $1064 \mathrm{~nm}$ laser radiation [12-14], or a pulsed tunable dye laser, which is cumbersome and not suitable for field study [15]. The cw-CRDS using narrow linewidth diode laser in visible $(690 \mathrm{~nm})$ [16] and near-IR (1550 and $1650 \mathrm{~nm})$ $[16,17]$ was also utilized for the aerosol extinction measurements. However, these setups required complex electronics to control the optical cavity length and to modulate the diode laser output in order to couple the $\mathrm{cw}$ diode laser radiation into the cavity. Only recently, an instrument utilizing multimode diode lasers was reported by Langridge et al. [28].

In this paper, a dual-channel multimode diode laser CRDS system is described for measurements of aerosol optical extinction. The utilization of the multimode, broadband diode laser (linewidth $\sim 0.2 \mathrm{~nm}$ ) allows for a simple on-axis injection of the laser radiation into the cavity, while it still has a high spectral resolution for studying the aerosol optical properties. The multimode diode laser is inexpensive and compact, suitable for portable instruments. Furthermore, the increasing availability of the diode lasers at various wavelengths will allow simultaneous multiple wavelength measurements of the optical properties of aerosols, which can help determine the dispersion in the refractive index with high accuracy. The dual-channel setup can monitor both background gas (with the aerosols removed by a particle filter) and background gas plus aerosols, allowing accurate determination of the aerosol contribution in ambient measurements. This proof-of-principle work demonstrates the utilization of the multimode diode laser in a simple, inexpensive, and portable CRDS instrument for both laboratory and field studies of aerosols.

\section{Experimental}

\subsection{The CRDS system}

In a typical CRDS setup, laser pulses are injected and trapped in an optical cavity composed of two high-quality mirrors (with typical reflectivity $R>99.9 \%$ ). In an empty cavity, the intensity of the trapped laser pulses decreases by a small percentage in each round trip due to mirror loss, decaying as an exponential function of time (with a decay time constant or ringdown time $\tau_{0}$ ). When an extinction aerosol sample is present in the cavity, its scattering and absorption increase the loss, which can be determined from comparing the empty cavity ringdown time $\left(\tau_{0}\right)$ and the one with the analyte $(\tau)[18,20]$ :

$$
\sigma_{\mathrm{e}}=\frac{L}{c l}\left(\frac{1}{\tau}-\frac{1}{\tau_{0}}\right)=\varepsilon Q_{\mathrm{e}} N,
$$

where $L$ is the cavity length, $l_{\mathrm{s}}$ is the sample path length, and $c$ is the speed of light. CRDS has the advantage of being insensitive to the fluctuation in laser intensity. It is a 
rapid, real-time technique, with individual ringdown events on the millisecond time scale. Another advantage is that the extinction aerosols can be removed from the air sample stream using a filter, and it is possible to obtain the optical extinction measurements without any calibration.

In this work, a small blue diode laser was incorporated as the light source in a portable dual-channel CRDS spectrometer (Figure 1). The optical output from the diode laser (Sharp, $20 \mathrm{~mW}$, multimode, $408.5 \mathrm{~nm}, \sim 0.2 \mathrm{~nm}$ FWHM linewidth) was amplitude modulated on and off with a rectangular-wave voltage signal at a repetition rate of $2 \mathrm{kHz}$ and a duty cycle of $50 \%$. The rising and fall time of the rectangular wave (and the corresponding rising time of the laser emission) is $\sim 0.8 \mu \mathrm{s}$, which is negligible to the measured ring-down time $(\sim 18 \mu \mathrm{s})$. With a beamsplitter and two turning mirrors, the laser beams were injected into two ringdown cavities.

Since the diode laser was multimode and broadband, the laser radiation can be directly coupled into and built up in the cavity, without any requirements for active matching of the laser modes to the resonant frequencies of the cavity or a scheme to increase the cavity mode density [23]. When the laser output was modulated off, the light intensity in the cavity decayed exponentially, providing the ringdown time measurements. The aerosol optical extinction can be obtained from the time constants with $(\tau)$ and without $\left(\tau_{0}\right)$ the aerosols present in the cavity using eq. (6). Two CRDS channels were used in the spectrometer: a reference channel monitored the gas-phase background (with the aerosols removed by a filter), and a sample channel measured the background plus the optical extinction of the particles. The difference between these two channels gave the aerosol optical extinction. This approach is particularly useful for the ambient measurements, in which the gas-phase background can be monitored and removed in real time, providing

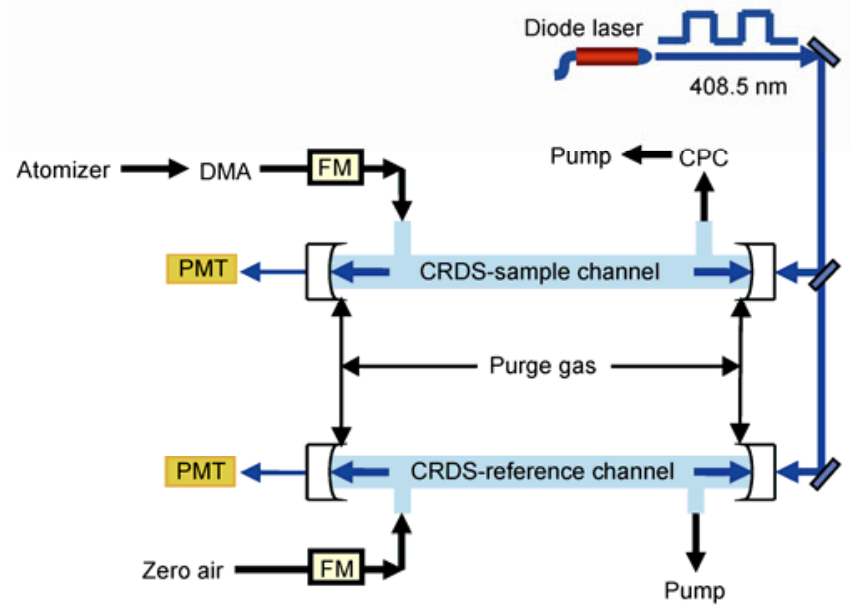

Figure 1 (Color online) Schematic diagram of the dual channel diode CRDS instrument for laboratory aerosol optical extinction measurements. FM, flow meter; DMA, differential mobility analyzer; CPC, condensation particle counter. enhanced sensitivity and time resolution. To demonstrate this approach, the diode laser was chosen at the near-UV wavelength $408.5 \mathrm{~nm}$ where the ambient $\mathrm{NO}_{2}$ absorbs [21]. For multiple wavelength measurements, diode lasers can also be selected to avoid or minimize the gas phase background.

Both cavity ringdown cells consisted of a pair of highreflectivity mirrors (Los Gatos, $R \approx 99.98 \%, 2.54 \mathrm{~cm}$ diameter, $1.0 \mathrm{~m}$ radius of curvature) separated at $86 \mathrm{~cm}$. The ringdown time constant was measured as $\sim 18 \mu$ s at 408.5 $\mathrm{nm}$. The mirrors were mounted on the home-made mirror mounts with purge volumes at both ends of the cell; the mirror mounts were connected to a $66 \mathrm{~cm}$ long glass tube (2.54 cm O.D.) through which the sample air flowed. The total cavity length $L$ includes the sample path length $l_{\mathrm{s}}$ and the lengths of the purge volumes. It has been shown in a previous study that the ratio of $L$ over $l_{\mathrm{s}}$ was independent of flow rate and in agreement with the measured physical dimensions of the cells [24]. Therefore, the cavity length $L=$ $86 \mathrm{~cm}$ and sample path length $l_{\mathrm{s}}=66 \mathrm{~cm}$ were used in eq. (6) without further calibration.

Tank air (Airgas) was used as zero air in this setup for both purge gas and aerosol carrier gas. Small purge flows (25 $\mathrm{mL} / \mathrm{min}$ zero air for each mirror) were introduced directly to the purge volumes in front of the mirrors for maintaining mirror cleanliness. Pressure and temperature were monitored independently for each cell. The light transmitted through the end mirror of each cavity was projected onto a photomultiplier tube module (Hamamatsu, H5783). Ringdown transients at a repetition rate of $2 \mathrm{kHz}$ were acquired with a PCI-based transient digitizer (Adlink, PCI-9846H/ 512,16 bits, $40 \mathrm{MHz}$ ). The 2000 ring-down traces were coadded and fit to a single exponential in every second. The data were collected and analyzed with a program written in house using the Adlink software development kit and the National Instruments Labview program.

\subsection{Laboratory aerosol generation and monitoring}

The laboratory aerosols used in this work were well characterized $\left(\mathrm{NH}_{4}\right)_{2} \mathrm{SO}_{4}$ particles, which have no absorption in the visible region. The $\left(\mathrm{NH}_{4}\right)_{2} \mathrm{SO}_{4}$ particles were generated by atomization of the $\left(\mathrm{NH}_{4}\right)_{2} \mathrm{SO}_{4}$ solution in distilled water using a homemade atomizer. The aerosol size distribution was varied by changing the concentration of the $\left(\mathrm{NH}_{4}\right)_{2} \mathrm{SO}_{4}$ solution.

Figure 1 shows the schematic diagram of the aerosol optical extinction measurements. The atomized aerosols were first dried by passing through a silicone gel dryer $(10 \mathrm{~cm}$ dia., $75 \mathrm{~cm}$ long) in the atomizer. The dried particles then flowed through a commercial differential mobility analyzer (DMA) (TSI, Model 3080) for selection in a nearly mono-dispersed size distribution. The size-selected aerosols had diameters ranging from 50 to $500 \mathrm{~nm}$ controlled by choosing the different voltages of DMA. The expected 
DMA distribution can be calculated using the width of the non-diffusive transfer function determined by Knutson and Whitby [29]. The actual transfer function of the DMA could be slightly wider due to particle diffusion, but that is neglected here [30].

The zero air sample containing the size-selected aerosols was drawn into the sample CRDS channel at a flow rate of $0.5 \mathrm{~L} / \mathrm{min}$ for the aerosol optical extinction measurements, while the reference CRDS cell was under zero air gas flow $(0.5 \mathrm{~L} / \mathrm{min})$ for background reference. A condensation particle counter (CPC) (TSI, Model 3772) was used to measure the particle concentration exiting the sample CRDS cell. The CPC detected particles from 10 to $1000 \mathrm{~nm}$ diameter. After the CPC, a pump was used to draw the sample at a flow rate of $0.5 \mathrm{~L} / \mathrm{min}$.

\subsection{Ambient measurements}

Ambient measurements were carried out for proof of principle in this work. A sampling inlet was positioned outside of the laboratory window. The ambient air sample from the common inlet was divided into the two CRDS cells. The sampling flow rate was maintained at $1.0 \mathrm{~L} / \mathrm{min}$ for each cell.

A $0.45 \mu \mathrm{m}$ Teflon particle filter was installed in front of the reference channel to remove the particles in the ambient air stream, and the background level (mainly from ambient $\mathrm{NO}_{2}$ ) [21] was obtained from the reference cell. The 0.45 Teflon filter has been demonstrated to efficiently remove all the particles in ambient air sample [21]. Meanwhile, the sample channel measured the background level plus the optical extinction of the particles. The difference between these two channels was the aerosol optical extinction. The aerosol sample exiting from the sample channel was also analyzed for its size distribution using the commercial scanning mobility particle sizer (SMPS), which is a built-in application combining the DMA and CPC.

\subsection{Extinction efficiency calculations}

The aerosol extinction efficiencies $\left(Q_{\mathrm{e}}\right)$ were calculated using the program MiePlot v4.1 (www.philiplaven.com) in this paper. This program is based on calculations and simulations using the Mie theory and Debye series. The program can compute the scattering intensity as a function of the scattering angle, wavelength, and radius and refractive index of the aerosols. It can produce the extinction cross sections and extinction efficiencies as functions of radius of the scattering sphere, size parameter and wavelength.

\section{Results and discussion}

\subsection{Comparison between the Mie theory calculations and the CRDS measurements}

The optical extinctions of the $\left(\mathrm{NH}_{4}\right)_{2} \mathrm{SO}_{4}$ particles were measured at $408.5 \mathrm{~nm}$ using CRDS and are compared with the predictions by the Mie theory. The optical extinction cross sections for aerosols of sizes between 50 to $500 \mathrm{~nm}$ are obtained. For example, Figure 2 shows sample data of experimentally measured extinction coefficients as a function of aerosol number density for the $\left(\mathrm{NH}_{4}\right)_{2} \mathrm{SO}_{4}$ aerosols of sizes of 310 and $500 \mathrm{~nm}$. The experimental data points are fitted with linear least-squares fitting. The slope represents the optical extinction cross sections (the product of $\varepsilon$ and $Q_{\mathrm{e}}$, eq. (2)). The Mie extinction cross sections are calculated using the refractory index of $\left(\mathrm{NH}_{4}\right)_{2} \mathrm{SO}_{4}$, which is $1.53+0.00 i$ near $400 \mathrm{~nm}$ at room temperature [31]. For several aerosols of sizes between 50 to $500 \mathrm{~nm}$, through the same analysis process, the extinction cross sections from the Mie theory and the CRDS measurements are summarized and compared for different aerosol sizes in Figure 3. In Figure 3 the Mie theory values are calculated using the selected aerosol sized set by DMA, counted by CPC and derived by eq. (2), while the experimental values points are derived using the CRDS measurements and eq. (6). As shown in Figure 3 , these two sets of extinction cross sections are in good agreement within $5 \%$ for aerosols ranging from 50 to $500 \mathrm{~nm}$ in diameter.

The experimental $Q_{\mathrm{e}}$ values of mono-dispersed spherical aerosols can be derived from the measured extinction cross sections in Figure 3 (eq. (6)). For spherical aerosols of diameter between 50 and $650 \mathrm{~nm}$, the $Q_{\mathrm{e}}$ values are calculated
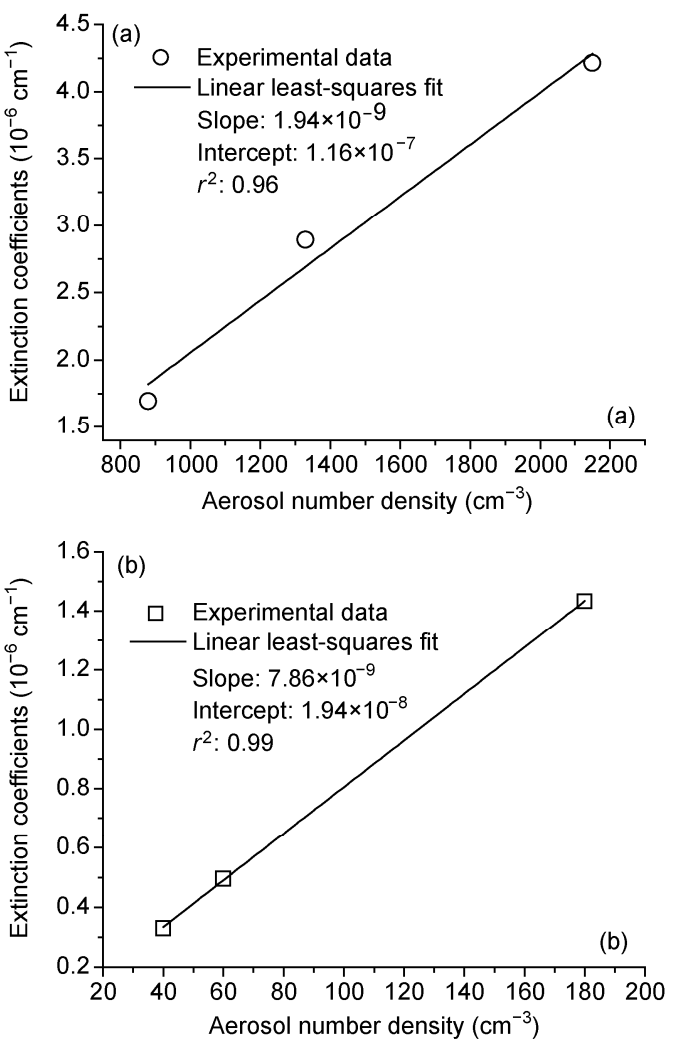

Figure 2 Measurements of extinction coefficient at $408.5 \mathrm{~nm}$ as a function of particle number density for particles with diameter of $310 \mathrm{~nm}$ (a) and $500 \mathrm{~nm}$ (b). 


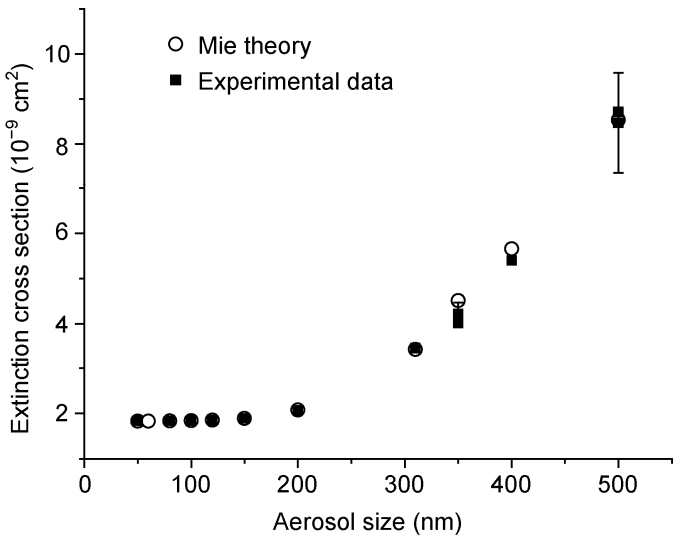

Figure 3 Comparison of extinction cross sections at $408.5 \mathrm{~nm}$ from the Mie theory and the CRDS measurements for aerosols of sizes from 50 to $500 \mathrm{~nm}$. The Mie theory points are calculated using the aerosol sizes selected by DMA and $n=1.53$ and $\lambda=408.5 \mathrm{~nm}$. The experimental points are derived using CRDS signals and eq. (6). The error bar represents $1 \sigma$ statistical uncertainty.

numerically using the Mie theory with the program MiePlot v4.1. Figure 4 shows the $Q_{\mathrm{e}}$ values of non-absorbing spherical $\left(\mathrm{NH}_{4}\right)_{2} \mathrm{SO}_{4}$ aerosols $(n=1.53$ at $\lambda=408.5 \mathrm{~nm})$ calculated from the Mie theory as a function of size parameter $x$ with a resolution of $x=0.1$. The extinction efficiency initially increases with the size parameter and reaches a maximum value of $\sim 4$ near $x=4$. There is a good correlation between the calculated and measured extinction efficiency values. The small difference between the calculations and measurements could be attributed to the actual shapes of the aerosol particles in the sample stream, which may not be completely spherical as assumed in the Mie theory calculations, or loss of the aerosols along the gas line before entering the CPC [15], even though the loss was limited by using a copper tubing.

\subsection{Ambient measurements}

As a test of the CRDS system, ambient measurements of optical extinction of aerosols were carried out in January 2011. The sample point was located outside of our laboratory building at the University of California, Riverside. The ambient air samples were drawn into a copper tubing through a hole in the windowsill of the laboratory and were then introduced into the CRDS cells. The copper tubing was used to limit the wall loss of the aerosol particles when sampled from the ambient air. The CRDS measurements were taken every $2 \mathrm{~s}$ in which 4000 ring-down traces were co-added. $\tau_{0}$ was obtained using zero air in both channels. In front of the reference channel, a $0.45 \mu \mathrm{m}$ Teflon particle filter was used to remove the particles in the ambient air sample. Another filter of the same type was used as an option in the sample channel when comparing the response of CRDS with and without the optical extinction of aerosols. It was assumed that the filter did not affect the composition of the air. The pressure drop over the filter was negligible.

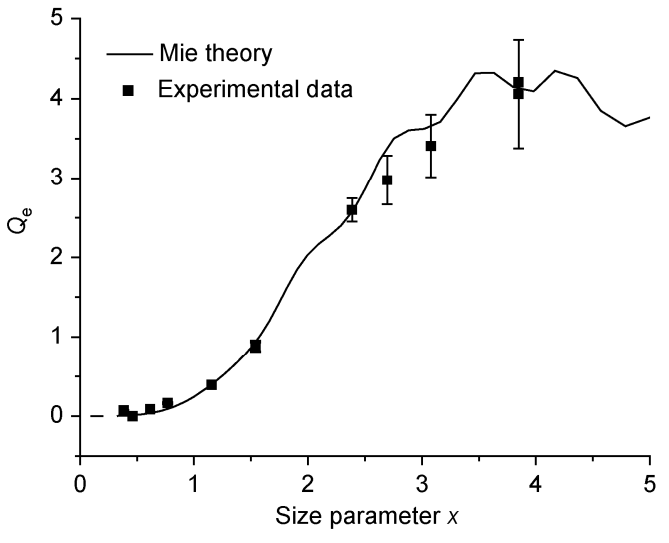

Figure 4 The extinction efficiency $Q_{\mathrm{e}}$ vs. the size parameter $x$ at 408.5 $\mathrm{nm}$. The experimental $Q_{\mathrm{e}}$ values are derived from the measured extinction cross sections in Figure 3 (eq. (6)), while those of the Mie theory are calculated using the program MiePlot v4.1 at a resolution of $x=0.1$ for $n=$ 1.53 and $\lambda=408.5 \mathrm{~nm}$ and the aerosol diameter of $50-650 \mathrm{~nm}$. The experimental error bar represents $1 \sigma$ uncertainty.

Since the Rayleigh scattering from small particles $(d<50$ $\mathrm{nm})$ and gas molecules are negligible when compared to the light loss from large aerosol scattering $(d>50 \mathrm{~nm})$ and $\mathrm{NO}_{2}$ absorption (here at $405.8 \mathrm{~nm}$ ), no corrections were made for changes in the Rayleigh scattering. The sensitivity of the CRDS system was calculated based on the zero air baseline noise. At an integration time of $10 \mathrm{~s}$, both channels can achieve the standard deviation precision of $0.24 \mathrm{Mm}^{-1}(1 \sigma)$. This detection limit is similar to those achieved in other CRDS instruments using pulsed lasers and modulated diode lasers $[3,8,9,28]$. The ambient measurement signals, however, were noisier due to the fluctuations from wind, temperature, pressure (not maintained at a constant in our setup), etc.

The size distribution of the aerosol sample monitored in the CRDS system was obtained by SMPS after CRDS (shown in Figure 5). The sampled ambient air had no aerosols larger than $300 \mathrm{~nm}$, either because of the clean ambient air in the winter and/or the loss of aerosols along the sampling line. Since under the current condition it is not possible

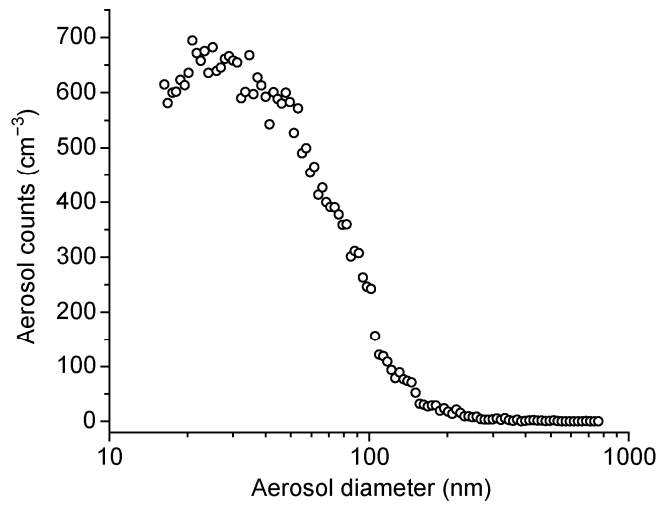

Figure 5 Ambient aerosol distribution obtained by SMPS through the sample system. There might be some wall loss. 
to obtain all the parameters of the ambient aerosols, such as their refractive index and shapes, it is difficult to accurately calculate the aerosol optical extinction from the Mie theory. If the ambient aerosols are assumed to be spherical and mainly composed of secondary organic aerosols (whose refractive index is in the range of 1.37-1.53 [32,33]), the total optical extinction can be estimated from the Mie theory by integrating all the aerosol optical extinction. The optical extinction coefficient for the aerosols shown in Figure 5 is estimated to be in the range of 1.6 to $2.8 \times 10^{-7} \mathrm{~cm}^{-1}$.

Figure 6 describes the CRDS measurement for ambient aerosol optical extinction coefficient from both the sample and reference channels. The reference channel sampled the ambient air with the particle filter removing all the aerosols, while the sample channel was tested with and without the particle filter. The gas phase species that absorbs significantly at $408.5 \mathrm{~nm}$ is $\mathrm{NO}_{2}$. The ambient $\mathrm{NO}_{2}$ was partially removed by the copper tubing, which was about 13 parts per billion ( $\mathrm{ppb}$ ) when reaching the CRDS cells, and was treated as the background (shown in the reference channel). The total extinction from $\mathrm{NO}_{2}$ plus aerosols was measured in the sample channel, and when the particle filter was in place there was a drop of the extinction coefficient of $\sim 1.3 \times 10^{-7}$ $\mathrm{cm}^{-1}$ from the ambient level, indicating removal of the contribution from the aerosol optical extinction. Furthermore, when the filter was in place in the sample channel, the signals from the sample channel and the reference channel were in agreement, indicating proper background measurements in the reference channel. The dual-channel approach

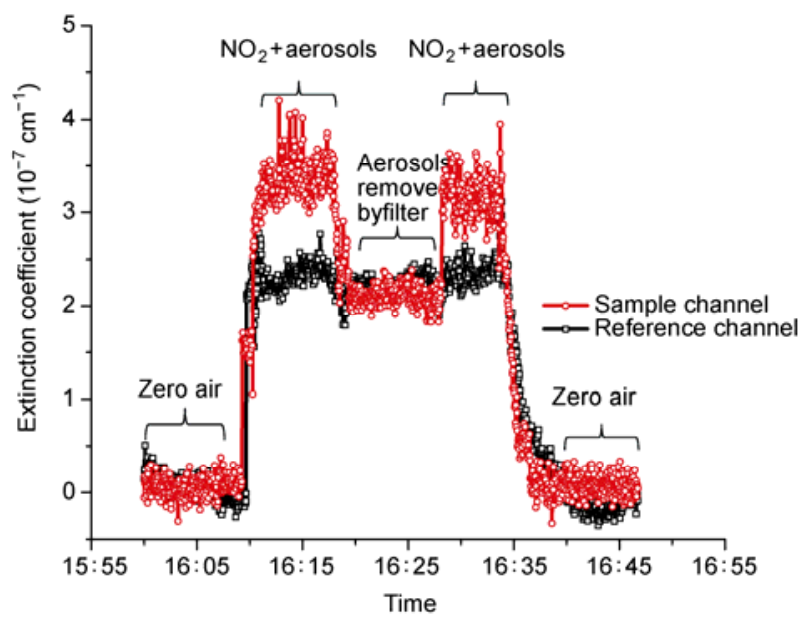

Figure 6 (Color online) CRDS measurement for ambient aerosol optical extinction coefficient in both the sample and reference channels. The system started with zero air for the baseline. At 16:10, the system drew in the ambient air; the sum of $\mathrm{NO}_{2}$ signal and aerosol optical extinction was obtained in the sample channel, while the reference channel monitored the $\mathrm{NO}_{2}$ background. The ambient $\mathrm{NO}_{2}$ was partially removed by the copper tubing, which was about $13 \mathrm{ppb}$ when reaching the CRDS cells, and was treated as the background (shown in the reference channel). At 16:17, a particle filter was installed in the sample channel to remove the aerosols, and the background $\mathrm{NO}_{2}$ level was measured in both channels. At 16:29, the total signal was recovered by removing the filter in the sample channel. At 16:35, the sampling was stopped and the zero air baseline level was obtained again. allows for continuous monitoring of the background and background plus aerosol extinction and thus real time removal of the background interference. Although the CRDS measurement $\left(\sim 1.3 \times 10^{-7} \mathrm{~cm}^{-1}\right)$ and the calculated optical extinction coefficient $\left(\sim 1.6 \times 10^{-7}\right.$ to $\left.2.8 \times 10^{-7} \mathrm{~cm}^{-1}\right)$ differ by a factor of up to 2 , this discrepancy could be due to the unknown properties of the ambient aerosols discussed before.

\section{Conclusion}

In this study, a broadband blue diode laser is used as the light source for direct coupling into the CRDS cells. The instrument is simple, inexpensive, and portable. The well-characterized laboratory generated $\left(\mathrm{NH}_{4}\right)_{2} \mathrm{SO}_{4}$ aerosols with different sizes in 50-500 $\mathrm{nm}$ are tested using the CRDS system, and the results are compared with the Mie theory calculations. Good agreement in $Q_{\mathrm{e}}$ within $5 \%$ is found. A dual-channel approach is applied for continuous monitoring of the background and background plus aerosol extinction signals, which allows for real time removal of background interferences. The sensitivity of the instrument can reach as low as $0.24 \mathrm{Mm}^{-1}(1 \sigma)$, which is suitable for the field study and ambient measurement.

Although only one diode laser wavelength is demonstrated in this proof-of-principle work, multiple wavelengths can be readily incorporated by simply replacing or adding other diode light sources. With the development of different types of diode lasers, using multiple wavelength light sources for aerosol optical extinction is possible. Measurements at multiple wavelengths can further provide information of the refractive index using the fitting of the extinction data. It can also provide an estimation of the single scattering albedo, which provides information on the extinction of light, including both scattering and absorption [34].

We thank Dr. D. Medina for assistance during several of the experiments. We thank Profs. D. Cocker and R. Atkinson for lending their equipments. This work was supported by the US National Science Foundation (CHE1214157) and W. M. Keck Foundation.

1 Seinfeld J H, Pandis S N. Atmospheric Chemistry and Physics: From Air Pollution to Climate Change. New York: John Wiley, 1998

2 Hinds W C. Aerosol Technology: Properties, Behavior, and Measurement of Airborne Particles. 2nd ed. New York: John Wiley, 1999

3 Sappey A D, Hill E S, Settersten T, et al. Fixed-frequency cavity ringdown diagnostic for atmospheric particulate matter. Opt Lett, 1998, 23: 954-956

4 Smith J D, Atkinson D B. A portable pulsed cavity ring-down transmissometer for measurement of the optical extinction of the atmospheric aerosol. Analyst, 2001, 126: 1216-1220

5 Bulatov V, Fisher M, Schechter I. Aerosol analysis by cavity-ringdown laser spectroscopy. Anal Chim Acta, 2002, 466: 1-9

6 Bulatov V, Khalmanov A, Schechter I. Study of the morphology of a 
laser-produced aerosol plume by cavity ringdown laser absorption spectroscopy. Anal Bioanal Chem, 2003, 375: 1282-1286

7 Pettersson A, Lovejoy E R, Brock C A, et al. Measurement of aerosol optical extinction at $532 \mathrm{~nm}$ with pulsed cavity ring down spectroscopy. J Aerosol Sci, 2004, 35: 995-1011

8 Spindler C, Riziq A A, Rudich Y. Retrieval of aerosol complex refractive index by combining cavity ring down aerosol spectrometer measurements with full size distribution information. Aerosol Sci Technol, 2007, 41: 1011-1017

9 Baynard T, Lovejoy E R, Pettersson A, et al. Design and application of a pulsed cavity ring-down aerosol extinction spectrometer for field measurements. Aerosol Sci Technol, 2007, 41: 447-462

10 Dinar E, Riziq A A, Spindler C, et al. The complex refractive index of atmospheric and model humic-like substances (HULIS) retrieved by a cavity ring down aerosol spectrometer (CRD-AS). Faraday Discuss, 2008, 137: 279-295

11 Dial K D, Hiemstra S, Thompson J E. Simultaneous measurement of optical scattering and extinction on dispersed aerosol samples. Anal Chem, 2010, 82: 7885-7896

12 Lang-Yona N, Rudich Y, Segre E, et al. Complex refractive indices of aerosols retrieved by continuous wave-cavity ring down aerosol spectrometer. Anal Chem, 2009, 81: 1762-1769

13 Freedman M A, Hasenkopf C A, Beaver M R, et al. Optical properties of internally mixed aerosol particles composed of dicarboxylic acids and ammonium sulfate. J Phys Chem A, 2009, 113: 13584-13592

14 Lang-Yona N, Abo-Riziq A, Erlick C, et al. Interaction of internally mixed aerosols with light. Phys Chem Chem Phys, 2010, 12: 21-31

15 Miles R E H, Rudic S, Orr-Ewing A J, et al. Measurements of the wavelength dependent extinction of aerosols by cavity ring down spectroscopy. Phys Chem Chem Phys, 2010, 12: 3914-3920

16 Strawa A W, Castaneda R, Owano T, et al. The measurement of aerosol optical properties using continuous wave cavity ring-down techniques. J Atmos Ocean Technol, 2003, 20: 454-465

17 Butler T J A, Miller J L, Orr-Ewing A J. Cavity ring-down spectroscopy measurements of single aerosol particle extinction. I. The effect of position of a particle within the laser beam on extinction. $\mathrm{J}$ Chem Phys, 2007, 126: 174302-174307

18 O'Keefe A, Deacon D A G. Cavity ring-down optical spectrometer for absorption measurements using pulsed laser sources. Rev Sci Instrum, 1988, 59: 2544-2551

19 Scherer J J, Paul J B, O'Keefe A, et al. Cavity ringdown laser absorption spectroscopy: History, development, and application to pulsed molecular beams. Chem Rev, 1997, 97: 25-51

20 Wheeler M D, Newman S M, Orr-Ewing A J, et al. Cavity ring-down spectroscopy. J Chem Soc Faraday Trans, 1998, 94: 337-351

21 Hargrove J, Wang L, Muyskens K, et al. Cavity ring-down spectroscopy of ambient $\mathrm{NO}_{2}$ with quantification and elimination of interferences. Environ Sci Technol, 2006, 40: 7868-7873

22 Osthoff H D, Brown S S, Ryerson T B, et al. Measurement of atmospheric $\mathrm{NO}_{2}$ by pulsed cavity ring-down spectroscopy. J Geophys Res, 2006, 111: D12305

23 Fuchs H, Dubé W P, Lerner B M, et al. A sensitive and versatile detector for atmospheric $\mathrm{NO}_{2}$ and $\mathrm{NO}_{x}$ based on blue diode laser cavity ring-down spectroscopy. Environ Sci Technol, 2009, 43: 7831-7836

24 Brown S S, Stark H, Ciciora S J, et al. Simultaneous in situ detection of atmospheric $\mathrm{NO}_{3}$ and $\mathrm{N}_{2} \mathrm{O}_{5}$ via cavity ring-down spectroscopy. Rev Sci Instrum, 2002, 73: 3291-3301

25 Ayers J D, Apodaca R L, Simpson W R, et al. Off-axis cavity ringdown spectroscopy: Application to atmospheric nitrate radical detection. Appl Opt, 2005, 44: 7239-7242

26 Medina D S, Liu Y D, Wang L M, et al. Detection of sulfur dioxide by cavity ring-down spectroscopy. Environ Sci Technol, 2011, 45: 1926-1931

27 Liu Y, Morales-Cueto R, Hargrove J, et al. Measurements of peroxy radicals using chemical amplification-cavity ringdown spectroscopy. Environ Sci Technol, 2009, 43: 7791-7796

28 Langridge J M, Richardson M S, Lack D, et al. Aircraft instrument for comprehensive characterization of aerosol optical properties. Part I: Wavelength-dependent optical extinction and its relative humidity dependence measured using cavity ringdown spectroscopy. Aerosol Sci Technol, 2011, 45: 1305-1318

29 Knutson E O, Whitby K T. Aerosol classification by electric mobility: Apparatus, theory, and applications. J Aerosol Sci, 1975, 6: 443-451

30 Olfert J S, Kulkarni P, Wang J. Measuring aerosol size distributions with the fast integrated mobility spectrometer. J Aerosol Sci, 2008, 39: 940-956

31 Toon O B, Pollack J B, Khare B N. The optical constants of several atmospheric aerosol species: Ammonium sulfate, aluminum oxide, and sodium chloride. J Geophys Res, 1976, 81: 5733-5748

32 Nakayama T, Matsumi Y, Sato K, et al. Laboratory studies on optical properties of secondary organic aerosols generated during the photooxidation of toluene and the ozonolysis of alpha-pinene. J Geophys Res-Atmos, 2010, 115: D24204

33 Redmond H, Thompson J E. Evaluation of a quantitative structureproperty relationship (QSPR) for predicting mid-visible refractive index of secondary organic aerosol (SOA). Phys Chem Chem Phys, 2011, 13: 6872-6882

34 Miles R E H, Rudic S, Orr-Ewing A J, et al. Influence of uncertainties in the diameter and refractive index of calibration polystyrene beads on the retrieval of aerosol optical properties using cavity ring down spectroscopy. J Phys Chem A, 2010, 114: 7077-7084

Open Access This article is distributed under the terms of the Creative Commons Attribution License which permits any use, distribution, and reproduction in any medium, provided the original author(s) and source are credited. 